\title{
Neurological Manifestations of HIV
}

\author{
Girish Modi, Andre Mochan and Mala Modi \\ Additional information is available at the end of the chapter \\ http://dx.doi.org/10.5772/intechopen.80054
}

\begin{abstract}
Neurological manifestations of human immunodeficiency virus (HIV) infection are common in certain regions of the world, notably Sub-Saharan Africa. The chapter highlights the neurotropism and neurovirulence of HIV underlying its direct neuropathology. The high frequency of neurological HIV disease is discussed in respect with the different viral clades. Sub-Saharan Africa is highlighted as bearing the brunt of the HIV pandemic. An approach to neurological HIV disease is given with a sensible classification system of manifestations and complications according to the level of immune suppression, primary HIV-related versus secondary opportunistic conditions, and other metabolic, drug induced, nutritional, or unrelated causes. Major manifestations of neuro-HIV are aseptic meningitis, HIV-associated neurocognitive disorders, HIV myelopathies and pediatric HIV-associated CNS disease; these are discussed in detail, and reference is made to the discrepancy of available data and literature between the so-called developed and developing countries. The role of antiretroviral treatment and its potential limitation in reaching the CNS compartment is stressed.
\end{abstract}

Keywords: HIV, neurology, pathophysiology, central and peripheral effects, special effects of HIV in childhood

\section{Introduction}

The human immunodeficiency virus (HIV) has a predilection to infect the nervous system. It is therefore neurotropic [1-3]. This correlates clinically with the fact that neurological symptoms occur commonly and during all stages of HIV infection [2,3]. Between 40 and 70\% of people infected with HIV will develop clinically symptomatic neurology, and at autopsy, $90 \%$ have neurological disease [2].

HIV-1, once it enters the human body, spreads hematogenously. The entry into the brain compartment is through blood-derived macrophages. The mechanisms are not completely understood 
but are proposed to involve cell trafficking across the blood brain barrier. The blood-derived infected macrophages or lymphocytes adhere to the vascular endothelium and then are thought to pass through it by EMPERIPOLESIS [2]. The trafficking-infected cell then transmits the virus to microglial cells or perivascular macrophages on the brain side of the blood brain barrier, a "Trojan horse" type of mechanism. The virus in these infected microglia then undergo productive replication and infects other microglia spreading the infection. Astrocytes are likewise infected but replication within these cells is incomplete or nonproductive and forms a reservoir. Neurons and oligodendrocytes are not directly infected by the virus and damage to these cells occurs by chemokines and cytokines released from the infected microglia and astrocytes [2,3].

The essential mediators of HIV-related CNS disease are the microglial soluble mediators, including quinolinic acid, TNF-alpha, IL-1 beta (Figure 1). Quinolinic acid binds to the NMDA receptor and increases calcium uptake with resultant activation of apoptotic mechanisms. TNF-alpha damages myelin and IL-1 beta stimulates astrocytes. Astrocytes produce nitric oxide and colony stimulating factors that feed back on microglia [2, 3].

The net result of this inflammatory cascade is an encephalitis, which is pathologically characterized by white matter pallor, neuronal loss, and astroglial reaction. This initiates and is the basis of primary HIV disease or as is commonly referred to as direct HIV infection of the nervous system.

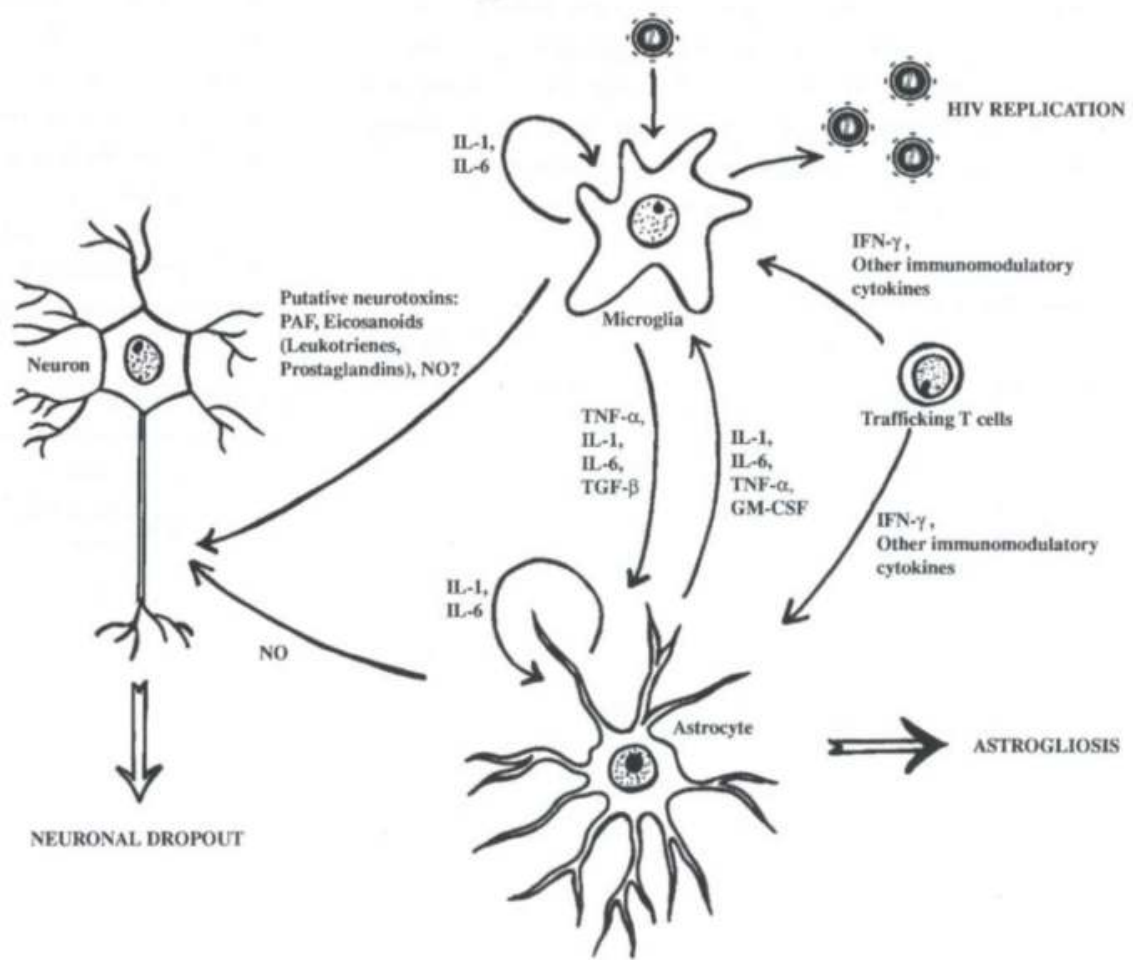

Figure 1. Neuropathophysiology of HIV infection in the Brain. 
The nervous system is also affected by HIV through indirect mechanisms that involve immunosuppression-related opportunistic disease and metabolic complications of systemic HIV infection and treatment with antiretroviral agents [1-3].

\section{Neurological disease classification}

The spectrum of HIV related or associated neurological disorders is broad and any part of the neural axis may be affected. Neurological complications of HIV are very stage-specific and relate to altered immune responses and deficiencies of cell mediated immunity-dysregulation of immunity [1, 2].

Metabolic diseases that result from dysfunction of other organ systems and toxic complications of drugs used to treat the HIV infection and its complications also cause neurological complications, especially in late stage HIV infection [1-3].

Dysregulation of immunity is caused by:

1. impairment of protective defenses with reduction in CD4 lymphocytes and macrophages. This occurs with late infection and is the most important determinant of neurological disease in HIV

2. elaboration of certain cytokines also determine neurological disease in late infection

3. autoimmune reactions in early HIV infection determine some neurological disorders

\subsection{Immune dysregulation}

1. Autoimmune disease (early and middle phases of HIV infection)

Acute phase encephalitis, neuropathies (AIDP)

Subacute and chronic inflammatory neuropathies

Acute disseminated encephalomyelitis (ADEM)

2. Immunosuppression: opportunistic infections/neoplasms (late phase HIV infection)

Cerebral toxoplasmosis

Primary CNS lymphoma (PCNSL)

CMV encephalitis

Cryptococcal meningitis

Progressive multifocal leukoencephalopathy (PML)

3. HIV driven

HIV-related neurocognitive disorders (HAND)

Distal sensory polyneuropathy 
Vacuolar myelopathy

HIV myopathy

\subsection{Secondary conditions}

1. Metabolic

Hypoxic encephalopathies

Narcotic overdose

Nucleoside neuropathies

Zidovudine myopathy

2. Psychiatric disorders

Reactive anxiety, depression

\section{Other}

Nutritional and metabolic disorders

Drug toxicity

Cerebrovascular complication

\section{Epidemiology of neurological HIV disease}

Studies on the prevalence of HIV among neurologic patients are sparse. The most often quoted study is the CDC study of 195,000 patients in 20 acute-care US hospitals, which found a seroprevalence of $0-13 \%$. This was highly correlated with the seroprevalence among all patients in the hospitals [2]. The study data was difficult to interpret as almost two-thirds of the HIV patients were previously undiagnosed. In a hospital-based study that audited HIV manifestations in medical inpatients in South Africa, the frequency of neurological involvement was $75 \%$, with $11 \%$ pure neurological disease, and $64 \%$ neurological and non-neurological disease combined [4].

The paucity of this type of data is in stark contrast to established data on the global prevalence of HIV from the Joint United Nations Programme on HIV/AIDS (UNAIDS). In 2016, the WHO estimated that 36.7 million people worldwide are infected with HIV. Sub-Saharan Africa bears the brunt of the HIV epidemic with 25.5 million infected individuals (a prevalence of $6 \%$ and $69 \%$ of all persons with HIV globally). Asia and the Pacific have 5.1 million infected people, and Latin America has 1.6 million infected people [5].

There are two principal subtypes of HIV, namely HIV-1 and HIV-2:

HIV-1, the predominant subtype is spread worldwide. HIV-2 was found predominantly in West Africa with scattered cases reported in the Americas and Western Europe. Both are associated with the clinical development of progressive immunological impairment with some differences in incubation and transmission properties. HIV-1 is the major cause of AIDS in humans [6]. 
There are several different HIV-1- clades based on phylogenetic data from the diverse HIV strains. Group M ("main") is responsible for the majority of infections worldwide, and is further divided into at least 10 distinct subtypes or clades A, B, C, D, F1, F2, G, H, J, and K. Group $\mathrm{O}$ ("out-group") is a relatively rare group currently found in Cameroon, Gabon, and France [6].

The different clades of HIV-1 are not distributed evenly throughout the world. Clade B dominates in North America and Europe. Clade C virus predominates in parts of Sub-Saharan Africa and Asia. Globally, clade $C$ virus is responsible for an estimated $50 \%$ of infections and is linked to the rapidly growing epidemics in Sub-Saharan Africa and some parts of Asia, mainly in India and China [6, 7].

The effect of the different clades in different populations on the pathological spectrum of HIV infection in these populations is not known. The majority of published data on HIVassociated neurological disease relates mainly to clade B, which is found in North America, Europe, and Australia. Information from regions where clade $C$ dominates is emerging and seems to indicate that there is no effect of the clade on the spectrum of neurological manifestations [4].

\section{HIV-associated neurological HIV disease}

\subsection{Aseptic meningitis}

\subsubsection{Introduction}

Aseptic meningitis is a clinicopathological syndrome, and the cardinal symptoms of which are headache, fever, and meningism. Pathologically, it is characterized by serous, nonpyogenic inflammation of the meninges. The defining cerebrospinal fluid (CSF) findings include a mononuclear pleocytosis, normal, or mildly raised protein and normal glucose levels [1]. Aseptic meningitis occurs with an annual incidence rate of $11-27$ cases per 100,000 population. The causes are mainly viral infections. Of these, enteroviruses (Echo and Coxsackie) make up $80 \%$ of cases followed by mumps, HSV-2, lymphocytic choriomeningitis, and adenovirus. Uncommon causes include infectious mononucleosis, cytomegalovirus (CMV), leptospirosis, HSV-1, mycoplasma, arboviruses (in epidemics mainly in the United States and Europe) and rarely during the icteric phase of infectious hepatitis. In the majority of instances, a causative agent cannot be established (exceptions include enteroviruses, mycoplasma, leptospirosis, and Lyme borreliosis) [1]. The aseptic meningitis in most of these conditions is a self-limiting illness and rarely is of sufficient severity to produce pathological changes in the brain that can be visualized with imaging modalities (CT or MRI).

It is important to recognize that an aseptic meningitis syndrome can occur in the course of other infectious and noninfectious inflammatory granulomatous and vasculitic and autoimmune illnesses. This is well described with respect to partially treated bacterial meningitis, so-called neighborhood infections, fungal, mycobacterial, spirochetal, and parasitic meningitis, malignant meningitis, and other noninfectious inflammatory diseases such as sarcoidosis, Behçet's disease, Wegener's granulomatosis, and granulomatous angiitis of the nervous system [1, 2]. 


\subsubsection{Aseptic meningitis in HIV}

Aseptic meningitis in HIV may be caused by HIV itself or by an opportunistic viral infection (CMV and JCV), mycobacterial infection, noninfectious inflammatory processes (immune reconstitution inflammatory syndrome, IRIS) or CNS neoplasia (lymphoma) [2]. In terms of direct infection, HIV has been identified in the CSF by polymerase chain reaction (PCR) or viral culture techniques throughout the course of HIV infection, and especially during late stage disease. Often despite extensive investigations, the causative infection or agent is not identified. In these latter situations, the aseptic meningitis is presumed to be due to HIV itself.

HIV-associated aseptic meningitis occurs in several different settings: at the time of seroconversion, during the course of the disease, and with highly active antiretroviral treatment (HAART) where it can be an IRIS-related manifestation $[2,3]$.

The aseptic meningitis in these HIV-related settings presents as an acute self-limiting illness (often with a cranial neuropathy, e.g., facial nerve palsy), an acute symptomatic meningitis or a chronic asymptomatic meningitis. Pleocytosis or elevated CSF protein has been described in almost two-thirds of asymptomatic HIV seropositive persons [2].

\subsubsection{Epidemiology}

Aseptic meningitis is the second commonest type of meningitis in HIV positive patients, the commonest being cryptococcal meningitis, which is described to affect between 5 and $7 \%$ of patients with AIDS [3].

The incidence or prevalence of aseptic meningitis in HIV cannot be accurately determined because it is most often asymptomatic (up to two-thirds of patients) and rarely symptomatic. Frequencies of $0.5-1 \%$ have been reported. No gender, ethnic, geographical, or clade-related differences have been described. The non-HIV conditions that can manifest as aseptic meningitis include cryptococcal meningitis, tuberculous meningitis, parasitic meningitis, and CNS neoplasms (lymphoma). Progressive multifocal leukoencephalopathy (PML) caused by JC virus can also mimic the CSF findings of an aseptic meningitis syndrome [2]. The relative frequencies with which these occur are related to the prevalence of infections in the environment and in this regard geographical and clade related differences have been described. Cryptococcal meningitis occurs with frequencies of $6 \%$ in South Africa (clade C), 3\% in India (clade C), 1\% in the United States (clade B), 1\% in Brazil (clade B), $7 \%$ in Uganda (clades A and D), and $2 \%$ in Thailand (clade E). Tuberculous meningitis, on the other hand, occurs in $6 \%$ of HIV-infected South Africans, 3\% of Indians, $1 \%$ of US citizens, $1 \%$ of Brazilians, $7 \%$ of Ugandans, and in $2 \%$ of Thai HIV-infected patients [4]. The frequencies of CMV and the other opportunistic pathogens causing HIV-associated aseptic meningitis are not well documented. The non-HIV viral infections described above that cause an aseptic meningitis syndrome have not been systematically studied in HIV-infected patients.

\subsubsection{Pathophysiology}

HIV enters the nervous system at any stage of infection in particular during the primary viremia that accompanies seroconversion. The mechanisms by which this process occurs are now better 
understood. Peripheral blood-infected monocytes carry HIV to the blood brain barrier and induce a macrophage tropism of tissue invasion. This HIV tropism for macrophages is determined by the V3 domain of the viral envelope glycoprotein. The infected macrophage releases adhesion molecules (intracellular or VCAMs), which cause adherence of the infected macrophage to the vascular endothelium. This triggers an immunological reaction involving inflammatory cytokines as well as matrix metalloproteinases that result in trafficking into the nervous system compartment. HIV may also penetrate the brain as free viral particles when there is a disrupted blood brain barrier. The subsequent events after invasion or trafficking are largely immunological and include activation of various cytokines with macrophage proliferation, microglial infection, and other processes that constitute so-called neurotropism and neurovirulence.

Meningeal inflammation occurs as a result of HIV breaching the meningeal blood-CSF barrier or from autoimmune processes causing an inflammatory response. Meningeal invasion occurs by hematogenous spread or via neurotropic mechanisms. Meningeal irritation produces reflex neck stiffness and causes headache and cranial nerve lesions.

\subsubsection{Clinical presentation}

The illness takes typically a biphasic course with initial nonspecific constitutional symptoms then followed by the classical features usually associated with meningitis, namely, headache, malaise, fever, neck stiffness, rigors, photophobia, nausea, and vomiting. Skin rash like the eruptions of varicella zoster (VZV) may appear concurrently. Other less common manifestations are cranial neuropathies, confusion, decreased level of consciousness and seizures.

\subsubsection{Investigations}

As described above, an aseptic meningitis syndrome is suspected when the CSF profile of a moderately raised protein with predominantly lymphocytic pleocytosis is identified. The crucial investigation is therefore CSF analysis. The subsequent investigations on the spinal fluid are performed to exclude conditions that mimic aseptic meningitis and in some instances to identify specific viral etiologies. In this regard, the following studies are useful:

Biochemistry, microscopy, and cellular counts

Gram stain, bacterial culture, and sensitivity

Acid-fast bacilli

India ink and cryptococcal antigen

Specific and nonspecific syphilis serology

PCR for HSV, VZV, Epstein-Barr virus (EBV), and CMV

The typical CSF profile in aseptic meningitis is that of a lymphocytic pleocytosis of less than 500 cells per $\mathrm{mm}^{3}$, normal or mildly elevated protein, normal glucose concentration, and negative bacterial antigen tests. Early CSF analysis may reveal a neutrophil predominance. An important differential diagnosis of this CSF constellation is partially treated bacterial meningitis especially in the presence of a history of recent antimicrobial therapy. Latex agglutination tests for bacterial antigens may be helpful in this setting. Aseptic meningitis 
due to noninfectious inflammatory diseases like sarcoid, Behçet's disease, uveo-meningeal syndromes usually have a more complicated course and always must be considered because they may respond to specific treatments $[2,3]$.

\subsubsection{Imaging}

CT or MRI may help to exclude suspected structural disease like parameningeal infectious foci, but is generally not necessary. A Chest X-ray may be indicated when tuberculosis is suspected as an underlying cause of aseptic meningitis.

\subsubsection{Treatment}

Management is symptomatic (fluids, analgesics, anti-inflammatories, antipyretics, antiemetics) with hospitalization often not required. If bacterial or partially treated bacterial meningitis is suspected, empiric antibiotics should be commenced. Acyclovir is used for HSV-1 or HSV-2, or severe EBV and VZV infections; antiretrovirals can be considered for HIV. Corticosteroids are not recommended because of their inhibitory effect on immune responses [1].

Hyponatremia as a consequence of infection-induced syndrome of inappropriate antidiuretic hormone secretion (SIADH) needs to be recognized and managed.

\subsubsection{Prognosis}

The outcome following aseptic viral meningitis is generally excellent with full recovery in 5-14 days after onset of symptoms. Rarely headaches, lightheadedness, and fatigue may persist for longer in some patients. The natural history of aseptic meningitis is determined by the natural history of the HIV infection and its effects on immunity. It is not clear whether the aseptic meningitis syndrome in HIV is a prelude to dementia, CNS neoplasia, or focal brain lesions $[1,2]$.

\section{HIV-associated neurocognitive disorder}

\subsection{Introduction}

Cognitive dysfunction in HIV infection is either due to the virus itself or caused by opportunistic disease resulting from progressive immunosuppression like CNS infections (cryptococcal meningitis, toxoplasma encephalitis, and progressive multifocal leukoencephalopathy) and neoplasia (lymphoma) [8].

Primary HIV-related neurocognitive disease is a result of direct infection by the virus, due to its predilection to invade (neurotropism) and cause disease (neurovirulence) in the CNS [9]. It is now identified worldwide as the commonest preventable and treatable neurocognitive illness in people below the age of 50 years [10].

Clinically, HIV neurocognitive illness comprises a spectrum ranging from mild asymptomatic neurocognitive impairment (ANI) through moderate mild neurocognitive disorder (MND) to 
severe HIV associated dementia (HAD) cognitive deficits [10]. The cardinal manifestations are a triad of cognitive, behavioral, and motor dysfunction.

\subsection{Epidemiology}

The HIV epidemic can be described in three phases. The first of these is the illness prior to the introduction of antiretrovirals, followed by the era of monotherapy with zidovudine (AZT), and now more recently, the era of highly active antiretroviral therapy (HAART) or combination antiretroviral therapy (cART).

Before the introduction of antiretrovirals dementia was a common manifestation of late disease occurring in over $50 \%$ of AIDS patients prior to death. That phase was characterized by a rapid turnover of prevalent dementia cases due to high incidence rates combined with high mortality rates resulting from AIDS-related complications (opportunistic infections and neoplasms). With the availability of monotherapy like zidovudine dementia rates and overall mortality decreased, but prolonged survival and incomplete recovery of prevalent cases has led to a relative increase of patients with the milder forms of HIV neurocognitive impairment (MCMD and NPI). This phenomenon has been enhanced more recently during the current era of HAART.

This scenario however only applies to the developed regions mainly of North America and Europe, where HAART is the standard of management. These regions are largely dominated by the clade B strain of HIV-1.

Clade C virus is responsible for an estimated 50\% of infections globally and associated with the epidemics in Sub-Saharan Africa, and parts of Asia, particularly India and China. How the different clades in different populations may influence the pathological and clinical spectrum of HIV infection is poorly documented [4]. Relatively little data is available on HIVrelated neurocognitive impairment from non-clade B regions. In these developing regions, HIV neurocognitive deficits were either poorly documented or thought to be a minor problem compared to the overwhelming burden of opportunistic infections. The apparently low prevalence of dementia was explained by possible under-diagnosis and underreporting, short life expectancy and short survival of HIV infected patients due to fatal opportunistic infections. Subsequent research has found the frequency to be higher than previously suspected with reported figures of 38\% in South Africa (clade C), up to 35\% in India (clade C), and 31\% in Uganda (clades A and D), indicating that the influence of clade subtype on the spectrum of cognitive dysfunction is probably minimal if at all $[4,11]$.

\subsection{Pathophysiology}

HIV enters the brain during the initial viremia following infection. This occurs through infected macrophage/monocyte lineage cells crossing the blood-brain barrier, the so-called "Trojan horse" mechanism or directly across the blood brain barrier [3].

In the brain parenchyma, mainly monocyte-derived cells (microglia and macrophages), and to a lesser extent astrocytes, can be infected by HIV. Penetration of microglial cells is via the cellular surface CD4 receptor in conjunction with the chemokine receptors CCR5 and CCR3 
leading to productive infection ultimately resulting in cell death. The exact mechanism of viral entry into astrocytes is unknown as these cells lack both CD4 and chemokine receptors. Following an initial productive phase of astrocyte infection, the virus enters a latent or restrictive, noncytopathic phase. This phase can persist long term and accounts for the latent virus escaping antiretrovirals currently in use.

Once the virus is within the brain parenchyma, it distributes selectively with the highest concentrations being found in the basal ganglia, subcortical frontal white matter and frontal cortex. This regionally preferential distribution within the brain may relate to viral entry through CSF pathways, to patterns of monocyte trafficking within the brain, or to possibly selective vulnerability of particular neuronal populations or anatomical brain regions. The neuropathology of these changes is white matter pallor, microglial nodules, multinucleated giant cells and gliosis; this pathological constellation is termed HIV encephalitis (HIVE). The extent of damage to synaptic and dendritic structures dominates over fairly mild neuronal loss. Histopathological damage and clinical severity correspond poorly, which implies that biochemical and immunological factors of host-virus interactions are determinants of the clinical dementia picture rather than structural changes. Neurotoxicity is thought to occur directly from viral proteins (gp 120, gp 41, tat, nef), or indirectly from macrophage factors (quinolinic acid, prostaglandins, leukotrienes), cytokines, and chemokines (TNF alpha, IL-1, IL-6, IL-10, interferons). In addition, disruption of the blood-brain barrier promotes access of neurotoxins from the systemic to the extracellular CNS compartment. Excitotoxicity through activation of NMDA receptors is the putative final common pathway resulting in neuronal dysfunction from disrupted cell energy metabolism and membrane integrity, with calcium influx leading to apoptosis [8, 11, 12].

\subsection{Clinical features}

HIV-associated cognitive impairment and dementia manifest over a period of weeks to months with the triad of cognitive decline, behavioral abnormalities, and motor dysfunction indicative of subcortical frontal lobe and basal ganglia involvement.

\subsection{Cognitive decline}

The early affected cognitive domains are verbal and visual memory retrieval, complex sequencing, and mental flexibility with the inability to sustain attention as the underlying phenomenon. The clinical effects are poor short-term memory, impaired concentration and executive dysfunction with mental slowing and flawed judgement. Patients present with increasing forgetfulness (appointments, medication schedules, and telephone numbers) and lose track of conversations and plots; the more complex daily tasks become difficult to complete timeously [11].

\subsection{Behavioral abnormalities}

These include lack of interest and drive, loss of libido, irritability, blunting of emotional responses, and waning engagement in work and hobbies, ultimately leading to social withdrawal, apathy, and inertia. Early subtle symptoms can easily be diagnosed as depression. Frank psychiatric presentations with delirium, mania, and psychosis can be presenting features in up to $10 \%$ of cases $[8,11]$. 


\subsection{Motor dysfunction}

Difficulties with fine finger movements and subtle balance problems are early motor features, manifesting with deterioration handwriting and a tendency to appear clumsy. Subtle gait difficulties may resemble the loss of quick righting reflexes as seen in patients with extrapyramidal disease. During these early stages, the neurological examination is normal except for mild slowing of repetitive movements (e.g., finger tapping), and increased deep tendon reflexes. Spasticity (especially of the lower limbs) with clonus, ataxia, frontal release reflexes, tremor, and sphincter disturbance evolve with progression of the disease. Seizures and myoclonus may appear late in the course. In advanced dementia signs of co-occurring myelopathy and/or peripheral neuropathy may contribute to the abnormal motor findings [8, 11, 12]. The presence of clear focal neurological signs like hemiplegia, hemianopia, hemisensory impairment, and cortical deficits such as apraxia, agnosia, or aphasia is suggestive of other or associated pathologies [11].

In advanced HIV-associated dementia (HAD), the picture becomes global with mutism, abulia, and incontinence followed by a vegetative state where all intellectual and social interaction is lost.

\subsection{Diagnostic criteria for HIV associated cognitive impairment}

Seropositivity for HIV

History of progressive alterations in cognition and behavior

Demonstrated impairment in at least two domains of neuropsychological performance

Absence of

Focal neurological signs

Intoxication or withdrawal (alcohol or other substance)

Metabolic derangement

CNS opportunistic infection or neoplastic lesions

Asymptomatic neuropsychological impairment (ANI), minor neurocognitive disorder (MND), and HIV associated dementia (HAD) form part of a continuum, where activities of daily living are unaffected in ANI and MND, and are impaired to varying degrees in HAD. Since there are no globally accepted measurements of a patient's degree of impairment in work, social, or other daily activities, determining this degree of impairment caused by cognitive dysfunction is largely based on clinical judgement [11].

HIV-associated neurocognitive impairment is a clinical diagnosis, made after exclusion of other potential causes. The presence of commonly occurring comorbidities like substance use disorders, major depression, and hepatitis B or C infection does not exclude the diagnosis [10].

\subsection{Investigations}

No single or combination of laboratory tests or parameters can reliably establish the diagnosis of HIV-associated cognitive impairment. Ancillary blood and CSF investigations and neuroimaging studies are necessary and useful to exclude other potential causes of cognitive 
changes in HIV infection. The main differential diagnostic considerations include delirium secondary to drugs and metabolic derangements, encephalopathies due to substance abuse or head injury, CNS opportunistic disease (meningitis and focal brain lesions), and primary psychiatric conditions. Hepatic and renal failure can cause metabolic encephalopathy directly or via impaired drug clearance.

\subsection{Blood tests}

Full blood count, electrolytes, urea and creatinine, liver and thyroid functions, Vitamin B12 level, syphilis serology, CD4 count and viral load should be evaluated.

\subsection{Cerebrospinal fluid}

This can be normal but is usually abnormal. Patients with HIV-associated cognitive impairment have CSF abnormalities, typical of a lymphocytic pleocytosis with mildly elevated protein and detectable viral RNA (aseptic meningitis syndrome). Similar abnormalities however can be found in the CSF of neurologically normal HIV patients and are thus nonspecific and unhelpful in confirming a diagnosis of dementia. CSF analysis helps to exclude other etiologies, in particular cryptococcal and tuberculous meningitis, neurosyphilis, CMV encephalitis, and PML $[2,3,11]$.

High CSF viral load titers loosely correlate with worsening cognitive performance in patients with advanced disease (CD4 counts below 200 cells $/ \mathrm{mm}^{3}$ ), but since the introduction of HAART and the resultant viral suppression attained by most patients, CSF viral load is no longer useful as a potential marker of CNS infection. Molecular CSF markers of immunological activation like beta2-microglobulin, neopterin, and quinolinic acid are not useful in routine clinical practice due to lack of sensitivity and specificity [11].

\subsection{Neuroimaging}

Structural imaging with computed tomography (CT) or magnetic resonance imaging (MRI) is integral to the diagnostic evaluation of patients with suspected HIV associated cognitive impairment ruling out any opportunistic processes. In HAD, cerebral atrophy with ex vacuo ventriculomegaly is the respective radiological finding. The subcortical tissue loss with resultant increase in ventricular size mirrors progressive clinical deterioration. On T2-weighted or T2 FLAIR MRI sequences this appears as patchy and later confluent high intensity white matter signal change, more prominently involving frontal white matter with a characteristic sparing of the subcortical U fibers [11].

Functional imaging techniques (magnetic resonance spectroscopy, MRS; single photon emission computed tomography, SPECT; and positron emission tomography, PET) remain research tools at this stage.

\subsection{Neuropsychological testing}

This type of testing, when available, can be used for screening purposes in high-risk asymptomatic or early symptomatic patients, and for follow-up evaluation in patients with established 
cognitive impairment. Useful neuropsychological tests include those that examine psychomotor speed, verbal and nonverbal learning, and sustained attention [10]. Performance in these tests is interpreted by comparing patients' results to a normative control database and can be influenced by differences in racial, ethnic, cultural, and social background. Appropriate normative standards are not available for large parts of the developing world. The value of standard neuropsychological testing in these regions is questionable [4].

\subsection{Treatment/management}

The use of HAART has led to a decreased frequency of HIV dementia. This is due to the effect of HAART improving cognitive performance in some patients with already established deficits and delaying or even preventing the onset of symptoms in others. HAART is therefore the suggested standard treatment for patients with HIV-associated cognitive impairment. Despite this, there are no specific consensus treatment guidelines. Current evidence and recommendations support the commencement of HAART at diagnosis of seropositivity. HAART should be initiated at the earliest stage of neurocognitive impairment irrespective of the immunological stage as the severity of the impairment at the initiation of HAART is the strongest predictor of persistent cognitive deficits.

CNS penetration of antiretrovirals across the blood-brain barrier has become an important consideration in the planned selection of drugs for CNS-targeted treatment. In this respect, protein binding capacities, lipophilic properties and CSF virological responses are the pharmacological parameters that determine which particular drugs achieve better bioavailability within the CNS compartment. Such putative neuro-active antiretrovirals would theoretically be superior in dementia-targeted treatment. However, clinical trials have failed to consistently confirm such a benefit [11].

Clinical trials of neuroprotective therapies like the NMDA antagonist memantine, and the antioxidant and selective monoamino-oxidase B inhibitor selegiline, targeting pathophysiological mechanisms beyond viral suppression, have to date not yielded conclusive results. Their use in routine clinical practice is not recommended, but remains a subject of further research.

Symptomatic treatment of depression, anxiety, psychosis or mania in patients with HIV neurocognitive impairment remains an integral part of their management.

\section{HIV-associated myelopathies}

\subsection{Introduction}

HIV-associated myelopathies are less frequent manifestations than encephalopathies. The etiologies of HIV myelopathy include mainly infections and neoplasms. Vacuolar myelopathy (VM) is a manifestation of primary HIV infection as is HIV transverse myelitis. Opportunistic infectious etiologies include CMV, HSV1 and 2, VZV, HTLV1, measles, JC virus, tuberculosis (TB), pseudomonas, syphilis, nocardia, cryptococcus, aspergillus, and Toxoplasma gondii. Neoplastic myelopathy in HIV occurs with primary CNS lymphoma (PCNSL), metastatic 
lymphoma, astrocytoma, and plasmacytoma. Vascular myelopathy is described in the context of necrotizing vasculitis and disseminated intravascular coagulation (DIC) [12, 13].

\subsection{Epidemiology}

Myelopathy in HIV/AIDS occurs with a frequency of 5-10\%, compared with HAD frequencies of $15-30 \%$ and distal sensory polyneuropathy (DSP) frequencies of $15-50 \%$. These are US based data; in the South African Black population the myelopathy frequency is 3\% [4]. Little data is available from elsewhere.

In the US, the vacuolar myelopathy accounts for $5-10 \%$ of HIV-related neurological disease (or 20-55\% of HIV myelopathy). VM accounts for $4 \%$ of HIV neurological disease in Japan (clade B), 1\% in Brazil (clade B), and 2\% in South Africa (clade C) [4].

The commonest cause of myelopathy in HIV in South Africa is TB (18-50\%) [4].

\subsection{Pathophysiology}

The pathological hallmark of VM is patchy vacuolization, occurring mainly in the thoracic region and predominantly affecting the lateral and dorsal columns. Axonal degeneration is a secondary phenomenon. There is no significant inflammatory infiltrate.

The exact pathogenesis of VM is unknown. HIV-infected macrophages, microglia, and astrocytes secrete myelin toxic immunoactive substances like TNF $\alpha$, IL 1, and 6. TNF $\alpha$ causes oligodendrocyte and myelin damage via reactive oxygen species. This oxidative stress to oligodendrocyte membranes causes increased consumption of antioxidants (e.g., glutathione) and methyl groups, which are essential in myelin maintenance. In HIV patients with VM, S-adenosylmethionine (SAM), the universal methyl group donor is decreased like in patients with vitamin B12 deficiency, accounting for the striking pathological similarities, namely the vacuolar change. It is postulated that cytokines released by HIV infected macrophages lead, via SAM depletion to a metabolic disorder that causes the white matter vacuolization in VM. Co-occurrence of SAM depletion and macrophage activation in immune suppressed HIV negative individuals (hematological malignancies, organ transplantation) can produce a clinically and pathologically identical myelopathy [12-14].

\subsection{Clinical features}

VM clinically manifests as a subacute, gradually progressive dorsolateral thoracic spinal cord syndrome presenting with spastic paraparesis, hyperreflexia, and extensor plantar responses. Sensory ataxia $(30 \%)$ and a co-existent distal sensory neuropathy $(53 \%)$ are present while a crisp sensory level is rare (13\%). Bladder sphincter disturbance can occur. VM has been observed to co-occur with HAD and DSP as a possible distinctive syndrome [4].

Tuberculosis can cause a transverse myelitis (often longitudinally extensive), spinal meningitis and Pott's disease of the spine leading to cord compression. The latter is characterized by back pain, spinal tenderness and fever are characteristic features. Presentation is with para- or tetraplegia. TB myelopathy is common in endemic areas and is not HIV stage specific [4]. 
HSV 1 and 2, CMV, and VZV are other viral myelitides associated with HIV infection.

Co-infection of HIV and HTLV-1 has been reported in HTLV-1 endemic regions.

Toxoplasma gondii uncommonly causes myelitis in isolation or in conjunction with focal cerebral lesions.

\subsection{Investigations}

CSF findings in VM are nonspecific and nondiagnostic (raised protein with lymphocytic pleocytosis). MRI in VM is often normal, but thoracic high signal changes during progression and cord atrophy in the chronic phase may be observed. Increased T2 cord signal, meningeal, and nerve root enhancement have been described in viral or post viral myelitides, in particular with CMV. In TB of the spine vertebral body and intervertebral disc involvement appears as low signal on T1- and high signal on T2-weighted images; irregular endplates and enhancing paraspinal collections have been documented. Lymphoma shows focal areas of low signal on T1 and high signal on T2 with patchy contrast enhancement.

\subsection{Treatment}

There is no treatment for VM. Unlike with HAD, VM has not shown to respond to HAART. Vitamin B12 supplementation, although theoretically beneficial, has not shown any effect. Symptomatic treatment of spasticity and sensory symptoms is pragmatically indicated.

CMV spinal cord disease can improve with the use of ganciclovir alone or in combination with cidofavir. Herpes simplex myelitis requires high dose intravenous acyclovir.

HTLV-1-associated myelopathy responds temporarily to intravenous corticosteroids.

TB spine responds well to standard treatment.

Early diagnosis of a Toxoplasma gondii myelitis and therapy with sulfadiazine and pyrimethamine or Bactrim produce a good response.

Primary CNS lymphoma may respond to chemotherapy.

\section{HIV associated CNS disorders: children}

\subsection{Introduction}

AIDS in pediatric medicine and child health has been recognized and described since the early 1980s after the identification of the virus itself. It is now a leading cause of childhood morbidity and mortality [15].

Pediatric HIV is mainly acquired through vertical mother-to-child transmission. This occurs either transplacentally during fetal development in utero; peripartum during passage of the fetus through the birth canal; or postnatally from contaminated breast milk. Other routes of 
infection include horizontal transmission through sexual abuse or transfusion of contaminated blood products. Adolescent HIV infection follows the same modes of transmission seen in adults, that is, sexual exposure and intravenous drug use.

Pediatric HIV-related CNS disease is primarily caused by the virus itself affecting all components of the neural axis, but with particular predilection for the brain. The clinical manifestations described below are thus varied with the commonest being progressive HIV encephalopathy (PHE). This selective vulnerability of the brain may in part be due to the effect of HIV on an immature brain (see below).

CNS opportunistic infections and malignancies on the other hand do not contribute significantly to HIV associated CNS disorders in childhood [15].

\subsection{Epidemiology}

The available data shows that approximately 2.3 million children worldwide are living with HIV/AIDS with nearly 2000 new infections and 1500 deaths occurring daily. These figures refer mainly to the developing world, and in particular Sub-Saharan Africa, dominated by the clade C strain of HIV. In these regions less than $10 \%$ of HIV positive pregnant women have access to appropriate measures to prevent mother-to-child transmission. In the developed world (clade B virus), pediatric HIV has ceased to be a significant problem as a result of effective use and delivery of antiretrovirals during pregnancy, elective cesarean section and infant formula feeding.

In the United States and Europe, highly active antiretroviral therapy (HAART) has reduced the rate of PHE from 9 to $35 \%$ in the early years of the HIV epidemic to $0-2 \%$ currently. Few studies from Sub-Saharan Africa report cognitive and motor developmental delay affecting $15-40 \%$ of HIV-infected children. There is no published data on the effects of HAART in this population. PHE occurs in 32-36\% of HIV positive children in Latin America. Opportunistic CNS infections were relatively frequent at $34 \%$ in a Brazilian hospital-based study and at $12 \%$ in a study from Argentina. The latter study documented a remarkable reduction of severity and frequency of PHE after the introduction of HAART [15].

\subsection{Pathophysiology}

The CNS is affected early during the course of HIV infection. Neuro-invasion and pathogenesis are similar to that described in adults with HIV associated cognitive impairment.

The interaction between fetal astrocytes and endothelial cells is central to the development of the blood-brain barrier (BBB). This happens in early gestation. HIV interferes with healthy $\mathrm{BBB}$ development by restricted or nonproductive $\mathrm{CD} 4$ receptor independent infection of the astrocytes. The consequently impaired or disrupted BBB has increased susceptibility to neuroinvasion. In the immature brain this leads to the encephalopathy (PHE). The restricted infection of astrocytes causes neuronal dysfunction via loss of supporting growth factors and impaired neurotransmitter re-uptake with resultant excitotoxicity. Macrophages, microglia, and multinucleated giant cells are involved in productive HIV infection with viral replication. The consequent inflammatory cascade leads to production of neurotoxic cytokines. Neuronal loss in PHE occurs from these processes as an indirect result of HIV infection. Active infection of neurons or neuronal progenitor cells remains controversial, but may occur in children at low levels [15]. 
The pathophysiological mechanisms in pediatric HIV-associated CNS disorders reflect the complex interactions between HIV a maturing brain.

\subsection{Clinical features}

\subsubsection{Progressive HIV encephalopathy (PHE)}

Clinically well-defined triad of:

1. Acquired microcephaly due to impaired brain growth.

2. Progressive motor dysfunction.

3. Loss, plateau or delay of neurodevelopmental milestones.

Acquired microcephaly: The diagnosis is made with stagnating or decreasing serial measurements of head circumference in children below 2 years of age. In the older child with closed skull sutures, the impaired brain growth correlates with parenchymal atrophy on neuroimaging.

Progressive motor dysfunction: The motor deficit in PHE children typically results from pyramidal tract abnormalities and presents with impaired fine motor function and ultimately loss of gross motor skills. Tone is often spastic. Motor milestones are either not achieved or can be lost. Extrapyramidal dysfunction with parkinsonian features of rigidity, drooling, and hypomimic facies may occur; cerebellar involvement is unusual. Advanced disease leads to a spastic, bedridden state.

These motor features are symmetrical. The occurrence of focal deficits should alert to possible underlying structural brain involvement by a mass lesion or infarction.

Neurodevelopmental decline: Neurodevelopmental deterioration involves global cognitive deficit with language and visuospatial, attention, concentration, and executive function problems. Delayed language milestones especially of expressive language often herald other cognitive and motor impairments. The delay is more easily observed in school-age children, but may often be attributed to other causes like nutritional, environmental, and psychosocial factors. Other symptoms include behavioral problems such as social withdrawal, apathy, mood disorders, and impulsiveness.

The clinical disease pattern of PHE varies widely with respect to age of onset, rate of progression, and domain(s) of functional impairment. Onset of PHE is most common in the first year of life with incidence rates of $9.9 \%$ in the first, $4.2 \%$ in the second year and less than $1 \%$ thereafter. Rates of progression vary widely with rapid decline over few months or a gradual deterioration. Early disease onset, especially when accompanied by advanced immune suppression predicts a more rapid and aggressive course with high mortality [15].

\subsubsection{Static encephalopathy}

A static encephalopathy with nonprogressive deficit or neurodevelopmental delay is also described. In contrast to PHE, there is no regression and there may be spontaneous 
improvement. This encephalopathy may be directly due to the HIV infection or secondary to other neurological insults, for example, premature birth, pre-natal exposure to toxins or infectious agents, genetic factors or head injury.

\subsubsection{CNS infections}

HIV positive children are prone to CNS infections caused by common and opportunistic organisms. Congenital CNS infections (toxoplasmosis, CMV) have been documented albeit infrequently. The commonest reported opportunistic infections (OIs) are CMV encephalitis, Candida albicans meningitis and micro-abscesses secondary to septicemia. OIs and their spectrum and occurrence in developing countries are not well described in the literature. Endemic infections like tuberculosis may play a bigger role than in developed countries [4]. Malaria and HIV co-infection may contribute to neurodevelopmental delay in affected children [15].

The frequency of OIs overall is low in children compared to adults, where reactivation of previously acquired infections (toxoplasmosis, JC virus) is common.

\subsubsection{Cerebrovascular disease:}

Strokes, both cerebral infarction and intracranial hemorrhage have been described in HIVinfected children. The frequency is lower than that seen in adults. Hemorrhage can occur into a tumor or be due to thrombocytopenia. Cerebral infarction may result from the vasculitis accompanying meningitis, or from cardio-embolic disease secondary to cardiomyopathy. HIV aneurysmal vasculopathy has been described in pediatric AIDS cases with fusiform aneurysms affecting the arteries around the circle of Willis.

\subsubsection{Seizures}

HIV infected children are more prone to seizures than their HIV negative counterparts. Seizures, especially if focal/partial in onset should prompt a search for underlying localized cerebral pathology.

\subsubsection{Neoplasms}

Primary CNS lymphoma and metastatic lymphoma to the CNS have been described. The clinical presentation may be with seizures, focal neurological signs, or deteriorating mental function.

\subsubsection{Myelopathy}

Spinal cord syndromes are rare in children and due to reactivated infections, for example, CMV. Vacuolar myelopathy, a frequent manifestation in adults with HIV is rarely seen in children. 


\subsection{Investigations}

\subsubsection{Cerebrospinal fluid}

The CSF findings in patients with PHE are often normal, even in florid disease. Equally common are nonspecific changes including a slightly raised protein and lymphocytic pleocytosis. Intrathecal antibody production, oligoclonal bands and other markers of immune-activation may be present in PHE children, but also in neurologically intact patients. HIV viral RNA is typically present in the CSF and may loosely correlate with PHE severity. Suppression of CSF viral load is utilized as a marker of response to treatment.

CSF findings can be helpful in establishing the diagnosis of certain common or opportunistic infections (pyogenic, tuberculous and cryptococcal meningitis; toxoplasmosis; CMV encephalitis) and neoplasm (CNS lymphoma).

\subsubsection{Neuroimaging}

Computed tomography (CT) scans of the brain reveal varying degrees of cerebral atrophy with corresponding ventriculomegaly and white matter hypodensities. Bilateral symmetrical basal ganglia calcifications are commonly seen. Magnetic resonance imaging (MRI) is sensitive to detect white matter changes, but not the calcifications. Serial imaging studies might assist to document progression, but quantitative and volumetric studies have not yet been standardized to be useful surrogate markers for early diagnosis or disease progression in clinical practice. Emerging functional neuroimaging techniques like magnetic resonance spectroscopy (MRS), functional MRI (fMRI), and positron emission tomography (PET) may become future tools in early disease detection and monitoring of disease progression or response to treatment.

\subsubsection{Psychometric testing}

Neuropsychological and neurobehavioral assessment tools can be useful to document the neurodevelopmental deficit and its progression, but require careful and skilled interpretation. Many factors other than HIV may influence a child's performance on testing. In addition, HIV positive children are more likely to be exposed to poor socio-economic circumstances and low levels of maternal education, and are more likely to suffer from compounding illnesses like birth asphyxia, anemia, and malnutrition leading to a multitude of biological and psychosocial confounders.

Standard psychometric testing remains to children in most developing countries. Such assessment tools still need to be validated in regions outside the US and Europe and in a greater variety of languages and cultural settings.

\subsubsection{Treatment/management}

Implementation of public health systems preventing mother to child transmission effectively can eradicate pediatric HIV altogether. 
Once PHE has set in HAART should be initiated. The ideal timing of commencement and choice of regimen is yet to be determined. However, this is not a cure as it fails to completely eradicate the virus from the CNS reservoir.

\section{Conclusion}

Neurological HIV disease remains a common and often serious reality, particularly in developing countries. Both adult and pediatric populations are at risk; it is important for the physician to be able to recognize and treat both the common and uncommon manifestations.

Opportunistic infections continue to dominate the picture in the developing world, where the locally endemic infections like tuberculosis stand out as underlying etiologies.

Despite advances in antiretroviral therapies the CNS remains at risk due to its sanctuary status protected by the blood brain barrier and the potential of viral escape in this compartment. However, antiretroviral treatment has started and will continue to moderate and improve neurological HIV disease.

\section{Author details}

Girish Modi*, Andre Mochan and Mala Modi

*Address all correspondence to: gmodicns@mweb.co.za

Division of Neurology, Department of Neurosciences, School of Clinical Medicine, Faculty of Health Sciences, University of the Witwatersrand, Johannesburg, South Africa

\section{References}

[1] Victor M, Ropper AH. Adams and Victor's Principles of Neurology. New York: McGrawHill; 2001

[2] Berger JR, Levy RM. AIDS and the Nervous System. Philadelphia: Lippincott-Raven; 1997

[3] Gendelman HE, Lipton SA, Epstein L, Swindells S. The Neurology of AIDS. New York: Chapman \& Hall; 1998

[4] Modi G, Hari K, Modi M, Mochan A. The frequency and profile of neurology in black south African HIV infected (clade C) patients - A hospital-based prospective audit. Journal of the Neurological Sciences. 2007;254:60-64

[5] Available from: www.unaids.org/en/resources/documents/2017/2017_data_book 
[6] Kandathil AJ, Ramalingam S, Kannangai R, David S, Sridaran G. Molecular epidemiology of HIV. The Indian Journal of Medical Research. 2005;121:333-334

[7] Bures R, Morris L, Williamson C, Ramjee G, Deers M, Fiscus SA, Abdool-Karim S, Montefiori DC. Regional clustering of shared neutralization determinants on primary isolates of clade $\mathrm{C}$ human immunodeficiency virus type 1 from South Africa. Journal of Virology. 2002;76:2233-2244

[8] Ances BM, Ellis RJ. Dementia and neurocognitive disorders due to HIV-1 infection. Seminars in Neurology. 2007;27:86-92

[9] Mcguire D, So YT. Neurological dysfunction: Overview. In: Cohen PT, Sande MA, Volberding PA, editors. The AIDS Knowledge Base. 2nd ed. Boston: Mass, Little Brown \& Co; 1994. pp. 5.6-1-5.6-2

[10] Antinori A, Arendt G, Becker TJ, Brew BJ, Byrd DA, et al. Updated research nosology for HIV-associated neurocognitive disorders. Neurology. 2007;69(18):1789-1799

[11] Clifford DB, Ances BM. HIV-associated neurocognitive disorder (HAND). The Lancet Infectious Diseases. 2013;13(11):976-986

[12] Tan SV, Guiloff RJ. Hypothesis on the pathogenesis of vacuolar myelopathy, dementia and peripheral neuropathy in AIDS. Journal of Neurology, Neurosurgery, and Psychiatry. 1998;65:23-28

[13] Shankar SK, Mahadevan A, Satishchandra P, Uday Kumar R, Yasha TC, Santosh V, Chandramuki A, Ravi V, Nath A. Neuropathology of HIV/AIDS with an overview of the Indian scene. The Indian Journal of Medical Research. 2005;121:468-488

[14] Gray F, Gherardi R, Scaravilli F. The neuropathology of the acquired immune deficiency syndrome (AIDS) - A review. Brain. 1998;111:245-266

[15] Van Rie A, Harrington PR, Dow A, Robertson K. Neurologic and neurodevelopmental manifestations of paediatric HIV/AIDS. European Journal of Paediatric Neurology. 2007;11:1-9 
